\section{THU0428 THE EFFECTS OF NEUROMUSCULAR ELECTRICAL STIMULATION ON STRENGHT, PAIN, AND FUNCTION IN INDIVIDUALS WITH KNEE OSTEOARTHRITIS: A SYSTEMATIC REVIEW WITH META-ANALYSIS}

Camila Cadena ${ }^{1,2}$, Vinícius Bispo ${ }^{3}$, Karenina Modesto ${ }^{1}$, Gerson Cipriano ${ }^{4}$, João Luiz Durigan ${ }^{4}{ }^{1}$ Universidade de Brasília, Faculdade de Educação Física, Brasília, Brazil; ${ }^{2}$ Tribunal Superior Eleitoral, Fisioterapia, Brasília, Brazil; ${ }^{3}$ CEFITE, Brasília, Brazil; ${ }^{4}$ Universidade de Brasília, Fisioterapia, Brasília, Brazil

Background: Even thought Neuromuscular electrical stimulation (NMES) has been widely used as a non-pharmacological intervention in patients with knee osteoarthritis $(\mathrm{OA})$ to improve muscle strength and function, prior research has shown conflicting results regarding NMES effectiveness in knee OA treatment.

Objectives: The aim of this systematic review and meta-analysis was to investigate the effectiveness of NMES in muscle strengthen, pain, and function in individuals with knee $\mathrm{OA}$.

Methods: Only randomized controlled trials (RCTs) were included in this study. Two authors independently performed the study selection. We use Pubmed, Embase, LILACS, PEDro and Cochrane Central Register of Controlled Trials as data sources. The main outcome evaluated was muscle strength. Function and pain were assessed as secondary outcomes using the Western Ontario Macmaster (WOMAC) questionnaire and Timed get up and go (TUG) test. The methodological quality was assessed using PEDro scale.

Results: A total of 23,215 were initially identified. After selection of titles and abstracts, studies were selected for the full-text analysis, ten studies were included with a total sample size of 622 patients. The methodological quality of the selected studies was moderate, with a mean score of 5.5 on a $0-10$ scale (PEDro). The following analysis were performed:

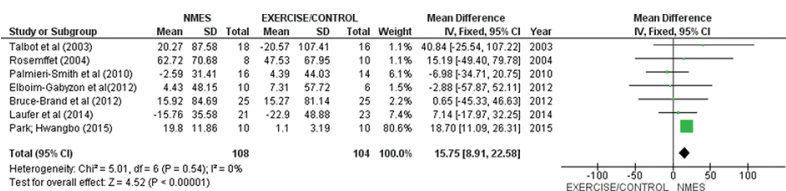

Abstract THU0428 - Figure 1. Meta-analysis of the effect of NMES on the increase of isometric muscle strength.

Legend: All measurements were standardized in $\mathrm{N} / \mathrm{m}$. A homogeneity in the data $\left(\mathrm{Chi}^{2}=5.01 ; \mathrm{I}^{2}=0 \%\right)$ was identified, with a significant difference in favor of NMES $(Z=4.52, p=0.01)$. NMES - Neuromuscular electro stimulation.

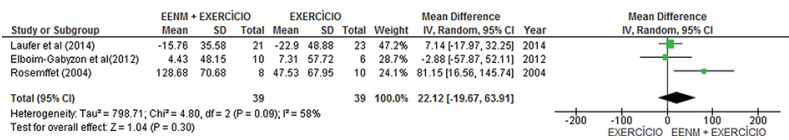

Abstract THU0428 - Figure 2. Meta-analysis of the effect of NMES associated with exercise vs conventional exercise in increasing isometric muscle strength.

Legend: A heterogeneity in the data was identified $\left(\mathrm{Chi}^{2}=4.80, \mathrm{I}^{2}=58 \%\right)$, with no significant difference in favor of NMES $(Z=1.04, p=0.30)$.

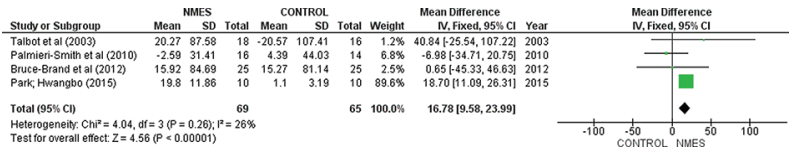

Abstract THU0428 - Figure 3. Meta-analysis of the effect of NMES vs control (No intervention) on isometric muscle strength

Legend: $A$ homogeneity in the data $\left(\mathrm{Chi}^{2}=4.04 ; \mathrm{I}^{2}=26 \%\right)$ was identified, with a significant difference in favor of NMES $(Z=4.56, p=0.01)$. $\mathrm{N}$

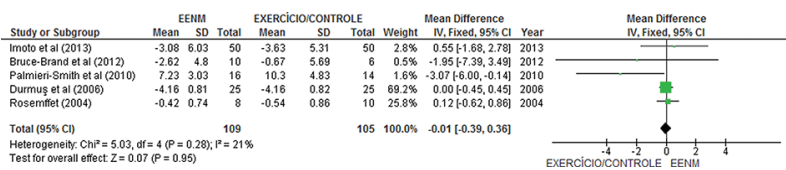

Abstract THU0428 - Figure 4. Meta-analysis of the effect of NMES on WOMAC pain
Legend: Data homogeneity $\left(\mathrm{Chi}^{2}=5.03 ; \mathrm{I}^{2}=21 \%\right)$ was identified, with no significant difference in favor of NMES $(Z=0.07, p=0.95)$. NMES Neuromuscular electro stimulation.

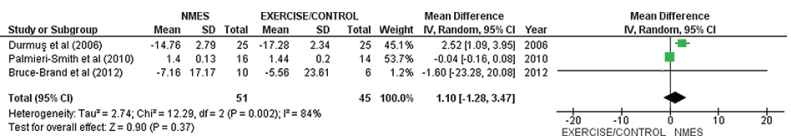

Abstract THU0428 - Figure 5. Meta-analysis of the effect of NMES on WOMAC function

Legend: Data homogeneity $\left(\mathrm{Chi}^{2}=12.29 ; \mathrm{I}^{2}=84 \%\right)$ was identified, with no significant difference in favor of NMES $(Z=0.90, p=0.37)$.

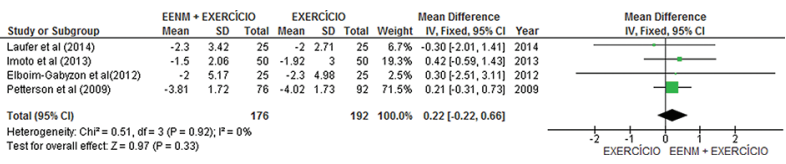

Abstract THU0428 - Figure 6. Meta-analysis of the effect of neuromuscular electrical stimulation on a secondary outcome: Timed Up and Go (TUG)

Legend: A homogeneity in the data $\left(\mathrm{Chi}^{2}=0.51 ; \mathrm{I}^{2}=0 \%\right)$ was identified with no significant difference in favor of NMES associated with exercise $(Z=0.97, p=0.33)$

Conclusion: NMES increased muscle strength in patients with OA compared to the active control group. However, the evidence to date did not demonstrate effects for pain control nor improvement in physical function.

\section{REFERENCES}

[1] Elboim-Gabyzon M, Rozen N, Laufer Y. 2013 Does neuromuscular electrical stimulation enhance the effectiveness of an exercise programme in subjects with knee osteoarthritis? A randomized controlled trial. Clinical Rehabilitation.27(3):246-257.

[2] De Oliveira Melo M, Aragão FA, Vaz MA. 2013 Neuromuscular electrical stimulation for muscle strengthening in elderly with knee osteoarthritis - A systematic review. Complementary Therapies in Clinical Practice.19(1):27-31

Acknowledgement: This research was supported by University of Brasília Disclosure of Interests: None declared

DOI: 10.1136/annrheumdis-2019-eular.3000

\section{THU0429 DISEASE BURDEN OF PERSONS WITH OSTEOARTHRITIS: RESULTS OF A CROSS-SECTIONAL ANALYSIS FROM A SURVEY LINKED TO CLAIMS DATA IN THE PROCLAIR PROJECT}

Johanna Callhoff ${ }^{1}$, Katinka Albrecht ${ }^{1}$, Imke Redeker ${ }^{2}$, Toni Lange ${ }^{3}$, Jens Goronzy ${ }^{3}$, Klaus-Peter Günther ${ }^{3}$, Angela Zink ${ }^{1,2}$, Jochen Schmitt ${ }^{4}$, Anne Postler ${ }^{3}$. ${ }^{1}$ German Rheumatism Research Centre, Epidemiology, Berlin, Germany; ${ }^{2}$ Charité University Medicine, Berlin, Germany, ${ }^{3}$ University Medicine Carl Gustav Carus Dresden, University Center of Orthopedics and Traumatology, Dresden, Germany; ${ }^{4}$ University Medicine Carl Gustav Carus Dresden, Dresden University Center for Evidence-Based Healthcare, Dresden, Germany

Background: Osteoarthritis $(\mathrm{OA})$ is a major reason for chronic pain, stiffness and functional limitation. While disease burden is frequently exam ined prior to surgery in knee and hip OA, burden in patients with unknown indication of joint surgery and the impact of polyarticular involvement are less often studied.

Objectives: The aim was to analyze factors associated with the burden of $\mathrm{OA}$, taking the pattern of joint involvement into account.

Methods: From a random sample of 8,995 persons with OA (ICD-10 codes M15 [polyarticular], M16 [hip] or M17 [knee]) from a large German statutory health insurance, 3,564 persons completed a questionnaire including the Western Ontario and McMaster Universities Osteoarthritis Index (WOMAC). Persons with knee, hip, concomitant knee and hip or polyarticular manifestation were compared concerning pain, stiffness, function, and impact on work/personal life, excluding asympomatic OA. Data were linked to dispensation records. The association of age, gender, BMI, symptom duration and WHO-5 with the WOMAC was assessed in multiple linear regression models.

Results: Persons with symptomatic knee $(1,130)$, hip (538), knee and hip (131) and polyarthritic (653) OA were included. Concomitant knee and hip OA was accompanied with the highest WOMAC values (mean 51), 
frequent impairment on work (66\%) and personal life (87\%) and the highest use of analgesics (60\% NSAIDs, $25 \%$ opioids and $40 \%$ others). In the regression analyses, BMI per 5 units and WHO-5 per 10\% worsening were associated with an increase in WOMAC values of 3-4 points, irrespective of the joint manifestations.

\begin{tabular}{|c|c|c|c|c|c|c|}
\hline & & & \multicolumn{4}{|c|}{ WOMAC } \\
\hline & Parameter & Reference & Polyarthrosis & Hip OA & Knee and Hip OA & Knee OA \\
\hline \multirow[t]{2}{*}{ Model 1} & Age & per 10 years & $0,7(-0,6 ; 2,0)$ & $0,7(-1,2 ; 2,6)$ & $2,0(-0,5 ; 4,4)$ & $-0,1(-3,5 ; 3,3)$ \\
\hline & Gender: Male & Female & $-3,8(-6,7 ;-1,0)$ & $0,8(-3,5 ; 5,0)$ & $-3,7(-8,1 ; 0,6)$ & $-0,5(-7,6 ; 6,6)$ \\
\hline tel $2^{*}$ & $\mathrm{BMI}, \mathrm{kg} / \mathrm{m}^{2}$ & per 5 units & $4,4(3,2 ; 5,5)$ & $3,1(1,1 ; 5,2)$ & $4,2(1,8 ; 6,6)$ & $4,1(0,6 ; 7,6)$ \\
\hline del $3^{*}$ & Symptom duratio & per year & $0,3(0,2 ; 0,5)$ & $0,4(0,2 ; 0,6)$ & $0,2(-0,0 ; 0,4)$ & $0,4(0,0 ; 0,8)$ \\
\hline tel $4^{*}$ & WHO-5 (0-100) & per $10 \%$ worseni & $8.4(3.8 ; 5.0)$ & $3.2(2.2 ; 4.1)$ & $4.1(3.3 ; 5.1)$ & $3.9(2.1 ; 5.9)$ \\
\hline
\end{tabular}

Table: Results of four separate multiple linear regression models with the WOMAC as the dependent variable Regression coefficients with $95 \%$ confidence intervals are shown.

*Model adjusted for age, gender. BMI: Body Mass Index, OA: Osteoarthritis, WOMAC: Western Ontario and McMaster Universities Osteoarthritis Score.

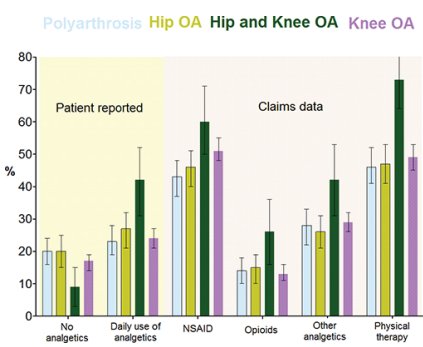

Abstract THU0429 - Figure 1. Patient reported use and claims data prescriptions of analgesics and physical therapy. NSAID: Non-steroidal anti-rheumatic drugs, OA: Osteoarthritis.

Conclusion: Disease burden is high in persons with concomitant knee and hip $\mathrm{OA}$, going along with frequent prescription of analgesics. The pattern of joint affection, BMI and depressive symptoms need to be considered when using the WOMAC as an outcome instrument.

Acknowledgement: This study was funded by the German Federal Ministry of Education and Research (01EC1405).

Disclosure of Interests: Johanna Callhoff: None declared, Katinka Albrecht: None declared, Imke Redeker: None declared, Toni Lange: None declared, Jens Goronzy: None declared, Klaus-Peter Günther: None declared, Angela Zink Speakers bureau: Speakers fees from AbbVie, Janssen, Pfizer, Roche, Sanofi, Jochen Schmitt: None declared, Anne Postler: None declared

DOI: 10.1136/annrheumdis-2019-eular.1202

\section{THU0430 PREPARING AN ORTHOPAEDIC CONSULTATION USING AN EHEALTH TOOL: A RANDOMIZED CONTROLLED TRIAL IN PATIENTS WITH HIP AND KNEE OSTEOARTHRITIS}

Aniek Claassen ${ }^{1}$, Henk J Schers ${ }^{2}$, Vincent Jjf Busch ${ }^{3}$, Petra Heesterbeek ${ }^{4}$, Frank van den Hoogen ${ }^{1,5}$, Thea Vliet Vlieland ${ }^{6}$, Cornelia van den Ende ${ }^{1,5} .{ }^{1}$ Sint Maartenskliniek, Department of Rheumatology, Nijmegen, Netherlands; ${ }^{2}$ Radboud University Medical Center, Department of Primary and Community Care, Nijmegen, Netherlands; ${ }^{3}$ Sint Maartenskliniek, Department of Orthopaedic Surgery, Nijmegen, Netherlands; ${ }^{4}$ Sint Maartenskliniek, Sint Maartenskliniek Research, Nijmegen, Netherlands; ${ }^{5}$ Radboud University Medical Center, Department of Rheumatology, Nijmegen, Netherlands; ${ }^{6}$ Leiden University Medical Center (LUMC), Department of Orthopaedics, Rehabilitation and Physical Therapy, Leiden, Netherlands

Background: Hip and knee OA patients who are referred to an orthopaedic surgeon often expect action to be taken. ${ }^{1}$ However, the majority who of those patients is not (yet) eligible for a joint replacement., ${ }^{2,3} \mathrm{We}$ hypothesized that a solid preparation using the eHealth tool is likely to streamline patients' expectations and increase satisfaction, irrespective of the outcome of the consultation.

Objectives: To evaluate the effect of a stand-alone mobile and webbased educational intervention (eHealth tool) compared to usual preparation of a first orthopaedic consultation of patients with hip or knee osteoarthritis (OA) on patients' satisfaction.

Methods: A two-armed unblinded randomized controlled trial involving 286 patients with (suspicion of) hip or knee OA, randomly allocated to either receiving an educational eHealth tool to prepare their upcoming consultation $(n=144)$ or usual care $(n=142)$. Satisfaction with the consultation on three subscales (range 1-4) of the Consumer Quality Index (CQI - primary outcome) and knowledge (assessed using 22 statements on $\mathrm{OA}$, range 0-22), treatment beliefs (assessed by the Treatment beliefs in OsteoArthritis questionnaire, range 1-5), pain medication use (yes/no), assessment of patient's involvement in consultation by the surgeon (assessed on a 5-point Likert scale) and patient satisfaction with the outcome of the consultation (numeric rating scale), were assessed.

Results: No differences between groups were observed on the 3 subscales of the CQI (group difference $(95 \% \mathrm{Cl})$ : communication 0.009 ($0.10,0.12)$, conduct $-0.02(-0.12,0.07)$ and information provision $0.02(-$ $0.18,0.21)$ ). Between group differences $(95 \% \mathrm{Cl})$ were in favour of the intervention group for knowledge $(1.4(0.6,2.2))$, negative beliefs regarding physical activities $(-0.19(-0.37,-0.002)$ and pain medication $(-0.30$ ($0.49,-0.01)$ ). We found no differences on other secondary outcomes. Conclusion: An educational eHealth tool to prepare a first orthopaedic consultation for hip or knee OA does not result in higher patient satisfaction with the consultation, but it does influence cognitions about osteoarthritis.

\section{REFERENCES:}

[1] Samsson KS, Bernhardsson S, Larsson ME. "Take me seriously and do something!" - a qualitative study exploring patients' perceptions and expectations of an upcoming orthopaedic consultation. BMC Musculoskelet Disord 2017 Aug 24;18(1):367

[2] Barten DJ, Smink A, Swinkels IC, Veenhof C, Schers HJ, Vliet Vlieland TP, et al. Factors Associated With Referral to Secondary Care in Patients With Osteoarthritis of the Hip or Knee After Implementation of a SteppedCare Strategy. Arthritis Care Res (Hoboken) 2017 Feb;69(2):216-25

[3] McHugh GA, Campbell M, Luker KA. GP referral of patients with osteoarthritis for consideration of total joint replacement: a longitudinal study. $\mathrm{Br} J$ Gen Pract 2011 Aug;61(589):e459-e468

Disclosure of Interests: None declared DOI: 10.1136/annrheumdis-2019-eular.2665

\section{THU0431 FREQUENCY OF TENDON INVOLVEMENT AND ITS EFFECT ON HAND FUNCTION IN HAND OSTEOARTHRITIS}

|rina Gess| ${ }^{1}$, Anna Vinatzer ${ }^{1}$, Gabriela Supp ${ }^{2}$, Michael Zauner ${ }^{1}$,

Michaela Loiskandl ${ }^{2}$, Martina Durechova ${ }^{1}$, Valentin Ritschl ${ }^{3}$, Josef S. Smolen ${ }^{1}$, Daniel Aletaha ${ }^{1}$, Peter Mandl' ${ }^{1}{ }^{1}$ Medical University of Vienna, Department of Medicine III, Division of Rheumatology, Vienna, Austria; ${ }^{1}$ Medical University of Vienna, Department of Medicine III, Division of Rheumatology, Vienna, Austria: ${ }^{3}$ Medical University of Vienna, Section for Outcomes Research, Vienna, Austria

Background: Tenosynovitis and consequent tendon damage are common findings in inflammatory arthritides. In contrast to rheumatoid arthritis (RA) and psoriatic arthritis, little is known about the frequency of tendon involvement in hand osteoarthritis ( $\mathrm{HOA})$ and the influence thereof on hand function. Ultrasound has been reported to have a high specificity in diagnosing tenosynovitis and tendon damage.

Objectives: We aimed to appraise the frequency of tendon involvement in HOA and to assess the agreement between ultrasound (US) and clinical diagnosis of tenosynovitis and tendon damage in HOA. In addition, we wanted to assess the influence of tendon involvement on hand function.

Methods: We included 73 patients with HOA in the study. Each patient underwent a clinical as well as a US examination of the 6 extensor tendon compartments and 6 flexor tendons of the hand (fig. 1). They were assessed for US signs of tenosynovitis and tendon damage as well as osteophytes (presence/absence) by a sonographer blinded to clinical information, as well as for clinical tendon involvement (presence/absence) by a biometrician blinded to the US results. Difference in frequency of sonographically detected tendon involvement between flexor and extensor tendons and between right and left hand were calculated by Chi-Square test. Osteophytes were also evaluated on standard radiographs. Agreement between US and clinical examination was calculated by Cohen's kappa. Hand function was quantified using the Score for the Assessment and Quantification of Chronic Rheumatoid Affections of the Hands (SACRAH) questionnaire as well as the Moberg pick-up test (MPUT). Correlation between MSACRAH and tendon involvement was calculated by Spearman's correlation.

Results: In 41 patients (56.2\%), at least one tenosynovitis was observed and in $8(11 \%)$ patients, at least one tendon damage was detected by US. Tendon damage was found more often in flexor tendons $(0.2 \%$ vs. $2.2 \%, p<0001$ ), while tenosynovitis was found more often in extensor 